\title{
Modifiye MWCNT/CuO Nanokompozitlerinin Optik Özellikleri ve Elektriksel İletkenlikleri Üzerine Non-iyonik Sürfektanın Etkisi
}

\author{
Filiz Boran $^{1 *}$ \\ ${ }^{1 *}$ Hitit Üniversitesi, Mühendislik Fakültesi, Kimya Mühendisliği Bölümü, Çorum, Türkiye, (ORCID: 0000-0002- 4315-9949), filizbektas@hitit.edu.tr \\ (1st International Conference on Applied Engineering and Natural Sciences ICAENS 2021, November 1-3, 2021)
}

(DOI: 10.31590 /ejosat.998137)

\begin{abstract}
ATIF/REFERENCE: Boran, F. (2021). Modifiye MWCNT/CuO Nanokompozitlerinin Optik Özellikleri ve Elektriksel İletkenlikleri Üzerine Non-iyonik Sürfektanın Etkisi. Avrupa Bilim ve Teknoloji Dergisi, (28), 306-311.

$\ddot{O} z$

Önceden sentezlenen $\mathrm{CuO}$ nanoparçacıklarının ve $\mathrm{MWCNT} / \mathrm{CuO}$ nanokompozitlerinin yapısal ve optik özellikleri üzerine farklı türev (polietilen glikol metil eter metakrilat) (PEGMEMA) ve moleküler ağırlıklara (Mw: 200, 2000 ve 5000) sahip polietilen glikol (PEG) noniyonik sürfektan kullanılmasının etkisi, X-ışını kırınımı (XRD), Enerji Dağılım X-Işınları analizi (EDS) ve UV-vis spektrofotometre ile kapsamlı bir şekilde araştırıldı. Bu örneklerin elektriksel iletkenlikleri iki nokta prob tekniği kullanılarak ölçüldü. Örneklerin X-ışını kırınım spektroskopisi verilerinden $\mathrm{CuO}$ nanoparçacıklarının ve MWCNT/CuO nanokompozitlerinin sirasıyla \%36,7-44,6 ve \%19,920,7 kristaliniteye sahip oldukları belirlendi. UV-vis spektrofotometre ölçümleri, non iyonik sürfektan kullanılması ile $\mathrm{CuO}$ nanoparçacıklarının ve MWCNT/CuO nanokompozitlerinin sırasıyla 4,24-4,31 eV ve 4,24-4,35 eV aralıklarında yüksek enerji bant aralıklarına sahip olduklarını göstermektedir. PEGMEMA kullanılması ile $\mathrm{CuO}$ nanoparçacıklarının ve MWCNT/CuO nanokompozitlerinin elektriksel iletkenlik değerleri sırasıyla $3,75 \times 10^{-5}$ den $7,93 \times 10^{-5} \mathrm{~S} / \mathrm{cm}$ 'e ve 55,75 'den $86,25 \mathrm{~S} / \mathrm{cm}$ 'e arttırılarak önemli ölçüde iyileştirildiği söylenebilir.
\end{abstract}

\section{Effect of Non-ionic Surfactant on Optical Properties and Electrical Conductivity of Modified MWCNT/CuO Nanocomposites}

\begin{abstract}
The effect of using polyethylene glycol (PEG) nonionic surfactant with different derivatives (polyethylene glycol methyl ether methacrylate) (PEGMEMA) and molecular weights (Mw: 200, 2000 and 5000) on the structural and optical properties of previously synthesized $\mathrm{CuO}$ nanoparticles and MWCNT/CuO nanocomposites was extensively investigated via X -ray diffraction (XRD), Energy Dispersion X-Ray analysis (EDS) and UV-vis spectroscopy. The electrical conductivity of these samples was measured using the twopoint probe technique. From the X-ray diffraction spectroscopy data of the samples, it was determined that CuO nanoparticles and MWCNT/CuO nanocomposites had crystallinity of 36.7-44.6\% and 19.9-20.7\%, respectively. UV-vis spectrophotometer measurements showed that $\mathrm{CuO}$ nanoparticles and $\mathrm{MWCNT} / \mathrm{CuO}$ nanocomposites had high energy band gaps in the range of 4.24-4.31 eV and 4.24$4.35 \mathrm{eV}$, respectively, with the use of non-ionic surfactant. It can be said that the electrical conductivity values of $\mathrm{CuO}$ nanoparticles and MWCNT/CuO nanocomposites were significantly improved by using PEGMEMA from $3.75 \times 10^{-5}$ to $7.93 \times 10^{-5} \mathrm{~S} / \mathrm{cm}^{2}$ and from 55.75 to $86.25 \mathrm{~S} / \mathrm{cm}$, respectively.
\end{abstract}

Keywords: $\mathrm{CuO}$, Electrical Conductivity, Energy band gap, Encapsulation, Crystallinity, MWCNT.

\footnotetext{
* Sorumlu Yazar: filizbektas@,hitit.edu.tr
} 


\section{Giriş}

Karbon nanotüpler (CNT'ler), eş merkezli silindirik düzlemlerin eksenel hizasında eğri $\mathrm{sp}^{2}$ hibritli karbon atomlarının düzenlenmesinden oluşan tüp şeklinde altıgen halkalara sahip nanoyapılardır. Bu tüplerin uzunlukları milimetrelere ulaşabilir ve "nanometre ölçeğinde" çapları vardır. Başta mükemmel mekanik ve elektriksel iletkenlik özellikleri olmak üzere, yüksek uzunluk/çap oranı, yüksek mukavemet, mükemmel akım taşıma kabiliyeti, geniş spesifik yüzey alanı, iyi parçacık emilimi ve yüksek kimyasal stabilitesi gibi olağanüstü yapısal, mekanik ve elektronik özellikleri nedeniyle CNT'ler birçok araştırmacının dikkatini çekmiştir [1-4].

Dikkate değer içsel özellikleri ve geniş potansiyel uygulamaları yelpazesi nedeniyle büyük ilgi gören CNT'lerin kullanımı için en ilginç firsatlardan biri nanokompozitlerde yatmaktadır [5]. CNT'ler, az miktarda CNT yüklemesi ile üretilen nanokompozitlerin mekanik özelliklerinde iyileşmenin yanı sıra önemli elektriksel iletkenlik sağlayabildiğinden önemli bir ilgi kazanmıştır [6].

İnorganik yarı iletken nanoyapılar, gaz algılayıcısı, inorganik kirleticilerin çıkarılması için katalizör, süperkapasitör ve saha emisyon cihazlarının yer aldığı mükemmel geniş uygulama yelpazesi nedeniyle CNT duvarının dekorasyonunda büyük dikkat çekmektedir [7]. Bu sebeple, son yillarda, fonksiyonel CNT'lerin $\mathrm{TiO}_{2}, \mathrm{ZnO}, \mathrm{ZnS}, \mathrm{In}_{2} \mathrm{O}_{3}, \mathrm{SnO}_{2}, \mathrm{CdSe}, \mathrm{CdS}$ ve $\mathrm{CuO}$ gibi yarı iletken metal oksitler ile modifiye edilmesine yönelik birçok çaba gösterilmiştir [7,8]. Bu yarı iletken nano yapılar arasından, foto iletken ve fotokimyasal özellikleri nedeniyle umut verici bir malzeme olan bakır oksit $(\mathrm{CuO}), 1,2 \mathrm{eV}$ 'luk dar bant aralığı ve $6,5 \mathrm{eV}$ çalışma fonksiyonu ile düşük elektriksel direnç değerleri olan p-tipi yarı iletkenlik sergiler [4,7]. $\mathrm{CuO}$, süperkapasitör, Liiyon batarya, fotokataliz, gaz sensörü, süperhidrofobik yüzeyler, saha emisyon cihazları dahil olmak üzere çeşitli alanlardaki çeşitli uygulamalarda dikkat çekmektedir [7]. Bu uygulamalar arasından ayrıca, $\mathrm{CuO}$ nanoparçacıkları, yüksek dislokasyon konsantrasyonları ve geniş yüzey alanları nedeniyle özel katalitik etki gösterdikleri için katalizör olarak da kullanılabilmektedir [9]. $\mathrm{Bu}$ sebeplerle, $\mathrm{CuO}$ nanoyapılarını $\mathrm{CNT}$ gibi yüksek iletkenlik malzemelerle dekore etmek anlamlıdır [2] ve $\mathrm{CuO}$ ile CNT kombinasyonu, üstün özelliklere sahip yeni birçok işlevli malzeme yaratacaktır [7].

CNT'ler içi boş tübüler morfolojisi iç kısımlarında çok çeşitli bileşiklerin kapsüllenmesine imkan sunarken, dış duvarların modifikasyonu kompozit malzemelerden enerji alanına, ilaç dağıtımına kadar uygulama alanlarını daha da genişletir. Ancak, tam potansiyellerinden yararlanmak için bazı engellerin aşılması gerekmektedir. Üretilen CNT'ler tipik olarak birkaç on mikrometreye kadar uzunluklara sahiptir ve güçlü hidrofobiklik sergiler, böylece işlenebilirliklerini sınırlar. Kısa ve fonksiyonel nano tüpler daha yüksek bir dağılabilirlik ve biyouyumluluk gösterir ve bu nedenle hedeflenen bazı uygulamalar için tercih edilir [10,11]. Fulleren C60 türevleri, su ve metal halojenür kristalleri gibi bir takım maddeler kanalların içine yerleştirilebilir. Kanallardaki uzamsal sınırlamalar nedeniyle CNT'nin içerisine yerleştirilen maddelerin özelliklerinde değişiklikler gözlenmektedir. Dolayısıyla, eğer metal katalizörler CNT'lerin içine getirilirse, yapıları ve elektronik özellikleri, CNT'lerin dış yüzeyinde bulunan katalizörlerden farklı bir şekilde etkilenebilir. Yapılan çalışmalar geçiş metallerinin redoks özelliklerinin CNT'ler içinde değiştirildiğini ve modifikasyon derecesinin e-ISSN: 2148-2683 nanotüplerin iç çaplarına bağlı olduğunu göstermektedir. Ayrıca, CNT'lerin içinde veya dışında tek tip bir boyuta sahip metal nanokatalizörlerin seçici olarak dağılması, şu ana kadar büyük bir zorluktur ve bu, katalizörlerin aktivitesini güçlü bir şekilde etkileyebilir [11].

Yukarıda belirtilenler ışı̆̆ında, bu çalışmada termal stres ile modifiye edilen MWCNT'lerin kanalları içerisine, farklı molekül ağırlıklarına sahip polietilen glikol (PEG) noniyonik sürfaktanları ve PEG'ün farklı bir türevi olan polietilen glikol metil eter metakrilat (PEGMEMA) kullanılarak sentezlenen $\mathrm{CuO}$ nanoyapılarının enkapsüle edilmesiyle önceden hazırlanan MWCNT/CuO nanokompozitlerinin [12] elektriksel iletkenlik özellikleri incelendi. Bu amaçla, öncelikle sentezlenen örneklerin kristallinite dereceleri XRD analiz verilerinden, tahmini enerji bant aralığı UV-vis spektroskopisinden ve MWCNT yapısına katılan $\mathrm{CuO}$ miktarı EDS analizi ile belirlendi.

\section{Materyal ve Metot}

$\mathrm{Bu}$ çalışmada kullanılacak olan örnekler, termal stres ile modifiye edilen MWCNT'lerin kanalları içerisine, farklı molekül ağırlıklarına sahip PEG noniyonik sürfektanları ve PEG’ün farklı bir türevi PEGMEMA kullanılarak sentezlenen $\mathrm{CuO}$ nanoyapılarının enkapsüle edilmesiyle önceki çalışmamızda hazırlanmıştır [12]. Bu prosedüre göre, ticari MWCNT'ler $600^{\circ} \mathrm{C}^{\prime}$ ye isıtılmış kül firınında 10 dakika bekletme ve 12 dakika oda sıcaklığında soğutma işemi üç kez tekrarlanarak termal stres ile işlevselleştirildi. Farklı türevi PEGMEMA ve farklı moleküler ağırlıklara (Mw: 200, 2000 ve 5000) sahip PEG noniyonik sürfektan kullanılarak sentezlenen $\mathrm{CuO}$ nanoparçacıklar $(\mathrm{CuO}$ PEGx, x: PEG'ün moleküler ağırlığı), ıslak emprenye tekniği ile modifiye MWCNT'nin kanallları içerisine kapsüllendi.

Sentezlenen örneklerin Enerji Dağılım X-Işınları analizi (EDS) ZEISS marka EVO 40 Model SEM (Taramalı elektron mikroskobu) cihazı kullanılarak yapıldı. Bu analiz ile yapıdaki $\mathrm{C}$ ve $\mathrm{CuO}$ miktarı yüzde cinsinden kalitatif olarak belirlendi. UVvis spektrofotometresi kullanılarak örneklerin süspansiyonlarının absorbansını incelemek için 200 ila $600 \mathrm{~nm}$ dalga boyu aralığında UV-görünür spektrumları alınmıştır. Örneklerin süspansiyonları pH 7'de 0,1 M fosfat buffer çözeltisinde hazırlanmıştır. Ayrıca nanokompozitlerinin kristalliği, X-1şını kırınımı (XRD) spektroskopisi verileri kullanılarak (Rigaku DMAX IIIC, $35 \mathrm{~kW}$, $15 \mathrm{~mA}, \mathrm{CuK} \alpha, 1,541871 \mathrm{~A}^{\circ}, 20: 5-80^{\circ}$, tarama hızı: $\left.2 \% \mathrm{dk}\right)$ belirlenmiştir. Nanokompozitlerin kristallik derecesi (\% XC) Eşitlik 1 ile hesapland.

$$
X c(\%)=\frac{A c}{A t} x 100
$$

Burada Ac ve At sırasıyla kristal tepe noktalarının altındaki ve tüm tepe noktalarının altındaki entegre alanı göstermektedir [13].

Sentezlenen örneklerin elektriksel iletkenliği, elde edilen numune peletinin, oda sicaklığında bir Thurlby elektrometresi ile standart iki nokta prob (two point probe) tekniği kullanılarak kapalı test odasına tabi tutulmasıyla ölçüldü. Elde edilen toz ürünlerin peletleri, 3 dakika boyunca $10 \mathrm{t}$ basınçta vakumlu pres altında tutularak hazırlandı [14-16]. Düzlem içi elektriksel iletkenlik aşağıdaki formülle hesaplandı:

$$
\sigma=\frac{1}{\rho}=\frac{1}{(R \times t)}(S / \mathrm{cm})
$$

Burada, $\rho$ elektriksel özdirenç, R elektriksel direnç ve t örnek kalınlığıdır [17]. 


\section{Araştırma Sonuçları ve Tartışma}

\subsection{Bulgular}

Sentezlenen $\mathrm{CuO}$ nanoparçacıklarının ve bu nanoyapıların modifiye MWCNT ile hazırlanan nanokompozitlerinin kristallik dereceleri (Xc) Tablo 1'de listelenmiştir. $\mathrm{CuO}$ nanoyapılarının farklı moleküler ağırlığa sahip PEG ve PEG'ün farklı bir türevi PEGMEMA kullanılarak sentezlenmesiyle kristallik derecelerinin bir miktar değiştiği görülmektedir. Çok düşük ve çok yüksek molekül ağırlığına sahip PEG kullanımı ile kristtallik dereceleri en yüksek değerlere ulaşmıştır. Farklı sürfaktanların kullanılması farklı boyutlarda $\mathrm{CuO}$ nanoyapılarının sentezlenmesiyle sonuçlanırken bu durumun kristallik derecelerini etkilediği Tablo 1 'den açıkça görülebilmektedir. Ancak, bu durum sentezlenen nanokompozitlerin kristallik derecelerini etkilemezken, MWCNT'nin yapıya eklenmesiyle kristallik dereceleri $\mathrm{CuO}$ nanoyapılarına göre azalmıştır.

Tablo 1. Örneklerin XRD verilerinden hesaplanan kristallik dereceleri

\begin{tabular}{l|c}
\hline \multicolumn{1}{c|}{ Örnekler } & Xc (\%) \\
\hline$C u O-P E G 200$ & 44,6 \\
\hline$C u O-P E G 950$ & 36,7 \\
\hline$C u O-P E G 2000$ & 39,3 \\
\hline$C u O-P E G 5000$ & 44,2 \\
\hline$C N T-M-C u O-P E G 200$ & 19,9 \\
\hline$C N T-M-C u O P E G 950$ & 20,6 \\
\hline$C N T-M-C u O P E G 2000$ & 20,7 \\
\hline$C N T-M-C u O P E G 5000$ & 20,6 \\
\hline
\end{tabular}

UV-Vis spektroskopisi, nanomalzemelerin tanımlanması için potansiyel bir ölçüdür [18]. Modifiye MWCNT yapısına katılan $\mathrm{CuO}$ nanoparçacıklarını daha fazla araştırmak için, hazırlanan örneklerin çözeltileri UV absorpsiyon spektrumu ile karakterize edilmiştir.

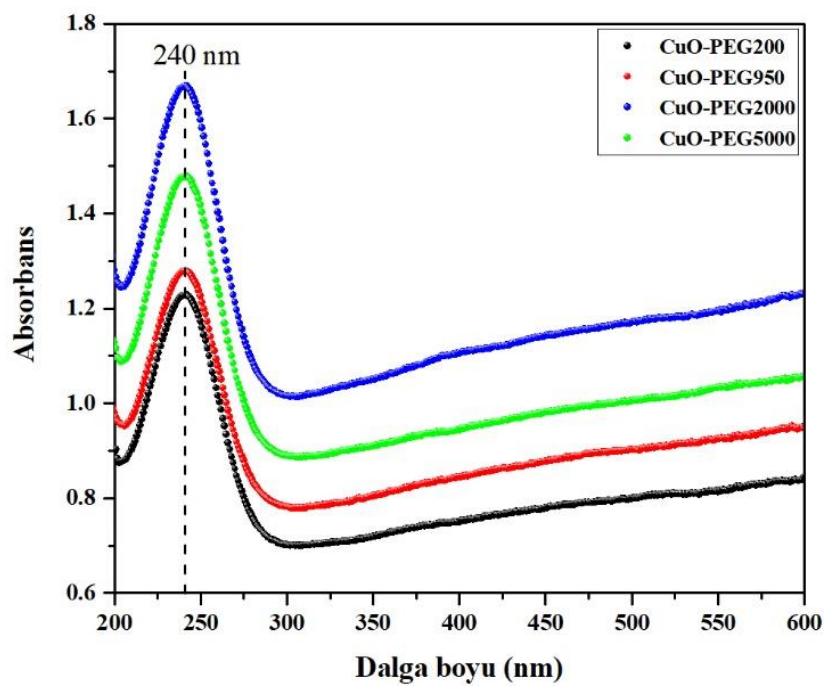

Şekil 1. CuO nanoparçacıklarının UV-vis absorpsiyon spektrumlarl

Fosfat tamponundaki farklı numunelerin UV absorpsiyon spektrumları Şekil 1 ve 2'de gösterilmektedir. CuO'lerin yapısından kaynaklanan yaklaşık $240 \mathrm{~nm}$ 'de bariz bir absorpsiyon zirvesi ortaya çıkmıştır. $\mathrm{CuO}$ nanoparçacıklarının sentezlenmesinde PEG ve PEGMEMA'nın kullanılmasının sadece absorbsiyon pikinin şiddetini değiştirdiği şekil 1'den anlaşılmıştır. Şekil 2'de gösterilen CNT-M-CuO örneklerinin absorpsiyon pikleri incelendiğinde ise, MWCNT'nin sulu ortamdaki zayıf stabilitelerinden dolayı UV-görünür bölgede herhangi bir tepe göstermediği [19] ancak $\mathrm{CuO}$ nanoparçacıklarına göre absorpsiyon pikinde kayma gösteren kompozitlerin CNT-M'nin yapıya ilave edilmesinden etkilendiği düşünülmektedir. $\mathrm{Bu}$ sonuca göre $\mathrm{CuO}$ nanoparçacıklarının MWCNT'lerin yapısına katıldığı söylenebilir.

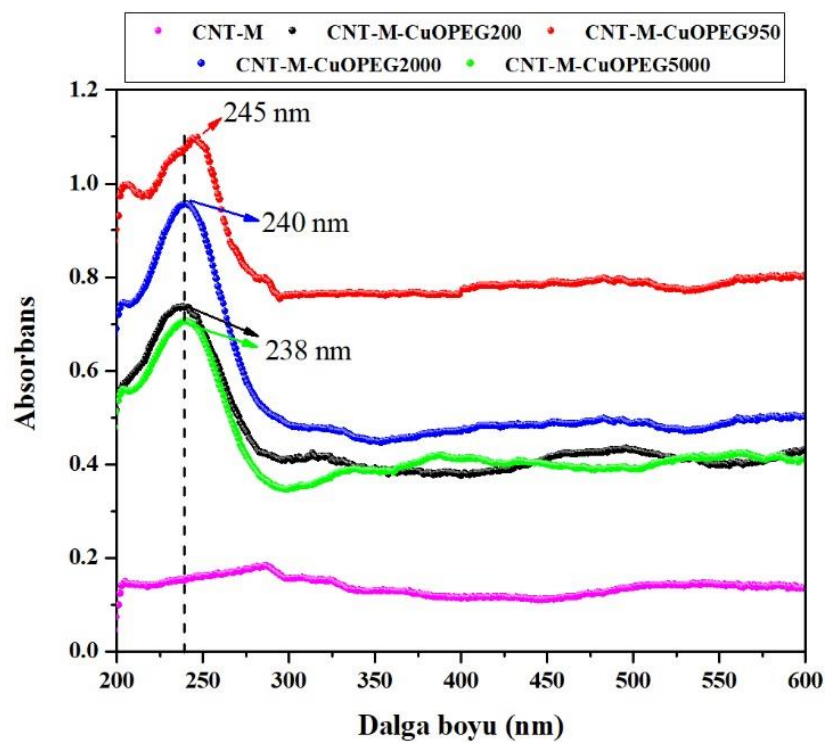

Şekil 2. CNT-M-CuO nanokompozitlerinin UV-vis absorpsiyon spektrumlarl

Sentezlenen $\mathrm{CuO}$ nanoparçacıklarının ve CNT-M-CuO nanokompozitlerinin optik enerji bant aralıkları, Kubelka-Munk denklemine (Eşitlik 3) göre $(\alpha h v)^{2}$,ye karş1 hv grafiğinin kullanılabileceği Tauc grafiği (Şekil 3-4) ile tahmin edilmiştir.

$$
(\alpha h v)^{2}=B(h v-E g)
$$

Burada, $\alpha$ absorpsiyon katsayıs1, h Planck sabiti, $v$ fotonun frekansı, Eg optik enerji bant aralığı ve B bir sabittir [20,21]. Örneklerin optik bant aralığı değerlerini belirlemek için deneysel eğrilere uyacak şekilde düz çizgiler çizilmiş ve bu çizgiler $(h v)$ eksenini kesecek şekilde uzatılmıştır [22]. Şekil 3'de gösterildiği gibi, farklı moleküler ağırlığa sahip PEG ve PEGMEMA kullanılarak sentezlenen $\mathrm{CuO}$ nanoparçacıklarının tahmini optik enerji bant aralığının 4,24 eV ile 4,31 eV aralığında değiştiği belirlenmiştir. Yapıya MWCNT'nin ilavesi ile nanokompozitlerin tahmini optik enerji bant aralıklarının 4,24 eV ile 4,35 eV aralığında değiştiği (Şekil 4) ve $\mathrm{CuO}$ nanoparçacıklarının optik enerji bant aralıklarını etkilemediği belirlenmiştir. Bu çalışmada farklı moleküler ağırlığa sahip PEG ve PEGMEMA kullanılarak sentezlenen $\mathrm{CuO}$ nanoparçacıklarının ve CNT-M-CuO nanokompozitlerinin optik özellikleri literatürle kıyaslandığında oldukça yüksek bant aralıklarına sahip oldukları görülmektedir [20,23-29]. Bu durum, nanomalzemelerin sahip olduğu boyuta bağlı optik özelliklerle sonuçlanan metal oksit yarı iletken malzemelerin nano boyutlu morfolojisinden kaynaklanan kuantum boyutu sınırlama etkilerine atfedilir [20,24]. 


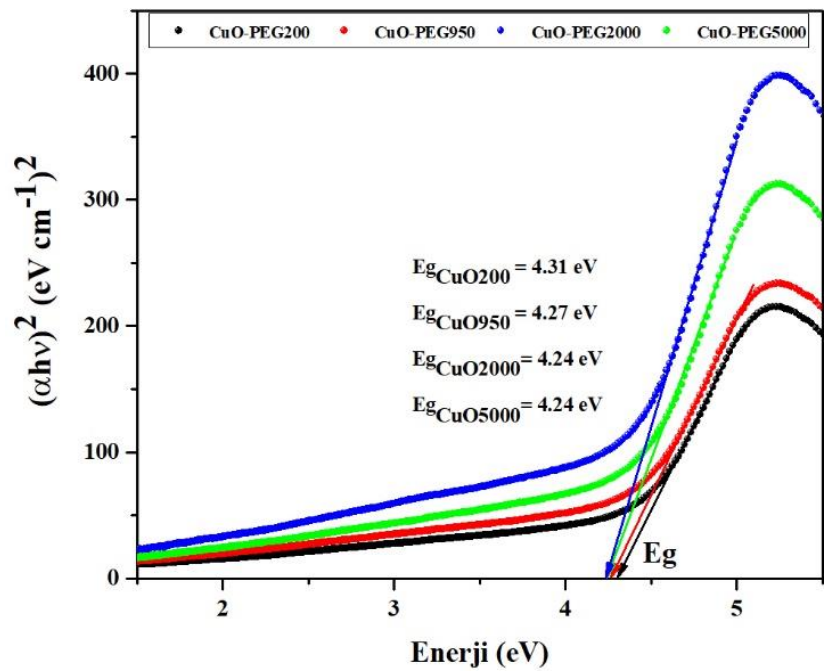

Şekil 3. CuO nanoparçacıklarının Kubelka-Munk (K-M) fonksiyonu ile Tauc ĕgrileri ve tahmini bant aralıkları

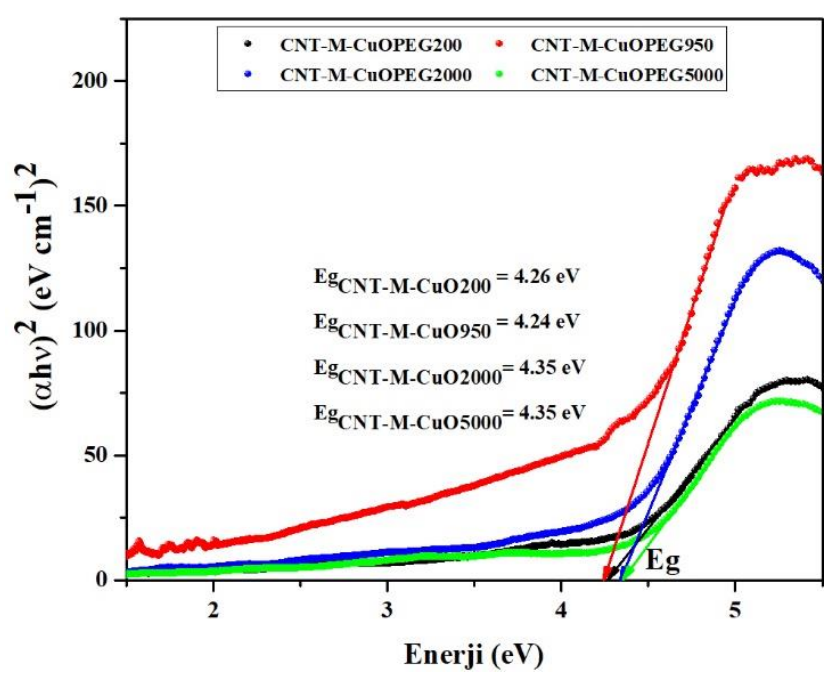

Şekil 4. CNT-M-CuO nanokompozitlerinin Kubelka-Munk $(K-M)$ fonksiyonu ile Tauc eğrileri ve tahmini bant aralıkları

Şekil 5'de önceki çalışmamızda verilen EDS sonuçlarından [12] elde edilen verilerle modifiye MWCNT yapısına katılan $\mathrm{CuO}$ miktarı, $\mathrm{Cu}$ yüklemesi hesaplanarak analiz edilmiştir [30]. modifiye MWCNT'nin yapısına katılan $\mathrm{Cu}$ miktarı farklı tür ve moleküler ağırlığa sahip PEG kullanılarak farklı boyutlarda elde edilen $\mathrm{CuO}$ nanoyapılarına göre değişmiş ve kullanılan sürfektanın moleküler ağırlığı arttıkça modifiye MWCNT'nin yapısına katılan $\mathrm{Cu}$ miktarının azaldığı görülmektedir. $\mathrm{Bu}$ sonuçlar önceki çalışmamızda verilen SEM ve R-TEM sonuçları [12] ile karşılartırıldığında en büyük parçacık boyutlarına sahip CuOPEG-200 nanoyapısı büyük boyutlarından dolayı modifiye MWCNT yüzeyinde çok fazla yı̆̆ılmalara ve yer kaplamalara sebep olmuştur. Bu nedenle modifiye MWCNT kanalları içerisine enkapsüle olamamış ve üzerine yerleşmiştir. Daha küçük parçacık boyutlarına rağmen modifiye MWCNT kanallarına az sayıda ve homojen olmayan şekilde enkapsüle olan CuO-PEG2000 ve CuO-PEG-5000 nanoyapılarının modifiye MWCNT yapısına daha az oranda katıldığı görülmektedir. Ancak PEGMEMA ile hazırlanan, önceki çalışmamızda verilen R-TEM sonuçlarına [12] göre en küçük ortalama parçacık boyutuna sahip CuO-PEG950 nanoyapısının uniform bir şekilde modifiye MWCNT kanallarına enkapsüle olması sebebiyle modifiye MWCNT yapısındaki $\mathrm{Cu}$ miktarı yüksek çıkmıştır.

e-ISSN: 2148-2683

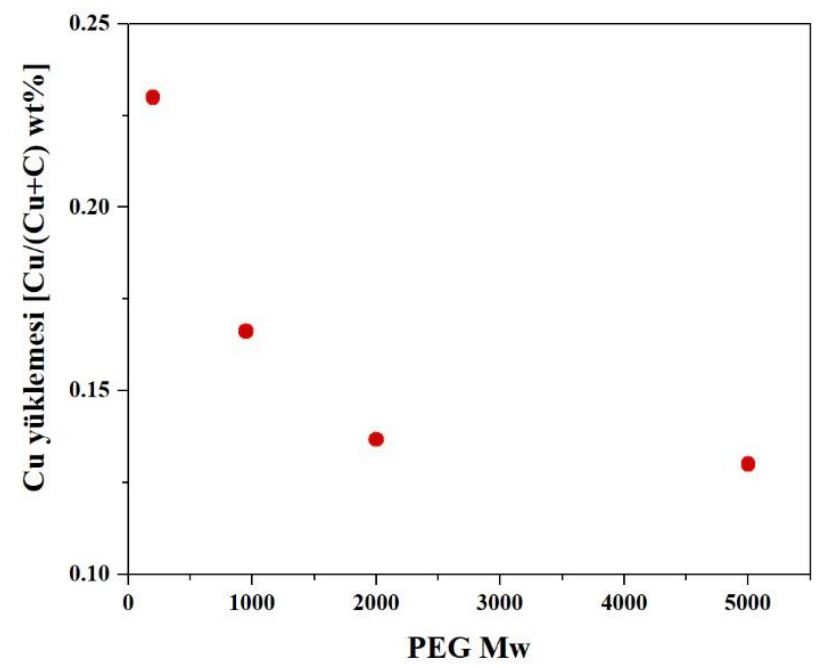

Şekil 5. EDS kullanılarak ölçülen modifiye MWCNT numunelerinde PEG'ün molekül ă̆ırlı̆̆ına göre bakır yüklemesi

Malzemelerin elektriksel iletkenliği, sentez parametreleri, kimyasal bileşimi, saflığı ve film kalınlığı, kristalit boyutu ve malzemelerin morfolojisi gibi yapısal faktörlerin değiştirilmesiyle etkilenebilir [16]. Dolayısıyla, nanokompozitlerin elektriksel iletkenlik gibi fiziksel özellikleri, CNT'lerin kompozitlerdeki dağılım durumuna güçlü bir şekilde bağlıdır. CNT'lerin yönlendirilmesi, CNT oryantasyonu yönünde nanokompozitlerin elektriksel iletkenlik özelliklerini iyileştirebilir [6]. Ayrıca, CNT'lerin elektronik iletim kabiliyetinin $\mathrm{CuO}^{\prime}$ dan daha üstün olduğu bilinmektedir. Dolayısıyla CNT'ler yüksek iletken kanal olarak ve $\mathrm{CuO}$ düşük iletken kanal olarak davranır. $\mathrm{Bu}$ nedenle, $\mathrm{CNT}-\mathrm{CuO}$ nanokompozitlerinin direnci büyük ölçüde $\mathrm{CuO}$ nanoyapılarının direncine de ayrıca bağlıdır [31].

Tablo 2. Örneklerin elektriksel iletkenlik değgerleri

\begin{tabular}{l|c}
\hline \multicolumn{1}{c|}{ Örnekler } & Iletkenlik (S/cm) \\
\hline Ticari $M W C N T$ & 89,25 \\
\hline Modifiye $M W C N T$ & 100 \\
\hline$C u O-P E G 200$ & $3,75 \times 10^{-5}$ \\
\hline$C u O-P E G 950$ & $7,93 \times 10^{-5}$ \\
\hline$C u O-P E G 2000$ & $5,43 \times 10^{-5}$ \\
\hline$C u O-P E G 5000$ & $4,2 \times 10^{-5}$ \\
\hline$C N T-M-C u O-P E G 200$ & 55,75 \\
\hline$C N T-M-C u O P E G 950$ & 86,25 \\
\hline$C N T-M-C u O P E G 2000$ & 75,75 \\
\hline$C N T-M-C u O P E G 5000$ & 71,5 \\
\hline
\end{tabular}

Ticari MWCNT, termal stres ile modifiye edilen MWCNT, sentezlenen $\mathrm{CuO}$ nanoparçacıkları ve bu nanoyapıların modifiye MWCNT ile hazırlanan nanokompozitlerinin oda sicaklığında ölçülen elektriksel iletkenlik değerleri Tablo 2'de listelenmiştir. $\mathrm{CuO}$ nanoyapılarının farklı moleküler ağırlığa sahip $\mathrm{PEG}$ ve PEG'ün farklı bir türevi PEGMEMA kullanılarak sentezlenmesiyle elektriksel iletkenlik değerlerinin değiştiği görülmektedir. Farklı sürfaktanların kullanılması farklı boyutlarda $\mathrm{CuO}$ nanoyapılarının sentezlenmesiyle sonuçlanırken bu durumun elektriksel iletkenliği etkilediği Tablo 2'den açıkça görülebilmektedir. $\mathrm{Bu}$ durum sentezlenen nanokompozitlerin elektriksel iletkenliğine de yansımıştır. Ticari MWCNT'nin termal olarak modifiye edilmesi, literatürde benzer sonuçlara 
rastlanıldığg gibi [32] MWCNT'nin elektriksel iletkenliğinin 100 $\mathrm{S} / \mathrm{cm}$ değerine artmasını sağlamıştır. Sonuç olarak, modifiye MWCNT kanallarının içerisine en iyi şekillde enkapsüle olduğu bildirilen CNT-CuOPEG950 nanokompoziti ile 86,25 S/cm değerinde en yüksek elektriksel iletkenliğe ulaşılmıştır.

\subsection{Tartışma}

Sentezlenen $\mathrm{CuO}$ nanoparçacıklarının modifiye MWCNT'lerin yapısına katıldığı XRD, EDS ve UV-Vis spektroskopisi analizi ile belirlenmiştir. UV-Vis spektroskopisi analiz sonuçları Ran ve arkadaşları tarafindan $\mathrm{Fe}_{3} \mathrm{O}_{4}$ nanoparçacıklarının MWCNT yapısına katıldığını gösteren benzer sonuçlar sergilemektedir [33]. Hussain ve arkadaşları, sentezledikleri $\mathrm{CuO}$ ve MWCNT ile kompozitinin tahmini optik band enerji aralıklarının sırasıyla 3,5 ve $3,7 \mathrm{eV}$ olarak belirlediklerini bildirmişlerdir [23]. Bu çalışmada sentezlenen örneklerin ölçülen tahmini bant aralığı enerjisi ise 4,24-4,35 eV aralığında değişmektedir. Farklı moleküler ağırlığa sahip PEG ve PEG'ün farklı bir türevi PEGMEMA kullanılmasının, literatüre göre daha yüksek değerlerde enerji band aralıklarına sahip $\mathrm{CuO}$ nanoparçacıklarının ve $\mathrm{MWCNT/CuO}$ nanokompozitlerinin sentezlenmesini sağladiğ görülmüştür.

Candemir ve Boran'nın yaptığı çalışmada molekül ağırlı̆̆ 4000 ve $8000 \mathrm{~g} / \mathrm{mol}$ olan PEG'lerin sentez ortamına ilave edilmesinin $\mathrm{CuO}$ nanoyapılarının morfolojisi ve elektriksel iletkenliği üzerine etkisi incelenmiştir. En yüksek 1,4090 $\times 10^{-7}$ S/cm elektriksel iletkenliğe $8000 \mathrm{~g} / \mathrm{mol}$ molekül ağırlıklı PEG'ün kullanılmasıyla ulaşıldığı bildirilmiştir [16]. Manjunath ve arkadaşlarının yaptığ 1 başka bir çalışmada polivinil alkol ile nanokompozitlerinin hazırlanması amacıyla sentezlenen toz formundaki $\mathrm{CuO}$ nanoparçacıklarının elektriksel iletkenlikleri $5,5 \times 10^{-6} \mathrm{~S} / \mathrm{cm}$ olarak bulunduğu rapor edilmiştir [34]. Bu çalışmada ise en yüksek $7,93 \times 10^{-5} \mathrm{~S} / \mathrm{cm}$ elektriksel iletkenliğe PEG'ün farklı bir türevi olan PEGMEMA kullanılarak sentezlenen $\mathrm{CuO}$ nanoyapısı ile ulaşılarak literatüre göre toz formundaki $\mathrm{CuO}$ nanoparçacıklarının elektriksel iletkenliği geliştirilmiştir. Ayrıca, elektriksel iletkenlik ölçüm sonuçları, PEG'in moleküler ağırlığının ve türevinin değişmesinin $\mathrm{CuO}$ nanoyapılarının ve modifiye MWCNT ile nanokompozitlerinin iletkenliğini arttırdığını göstermiştir.

$\mathrm{Bu}$ çalışmanın sonuçlarının, sayısız kullanım için MWCNT bazlı yarı iletken metal oksit nanokompozitlerin tasarımına katkıda bulunabileceğine inanılmaktadır.

\section{Sonuç}

Optik özellikleri araştırmak için UV-vis spektroskopisi kullanıldı ve UV-vis verileriyle ölçülen tahmini bant aralığ enerjisi 4,24-4,35 eV'dir. XRD verilerinden elde edilen kristalinitesindeki azalma, absorbsiyon bandında gözlenen kayma ve bant aralığı enerjisinde gözlenen değişiklikler, $\mathrm{CuO}$ nanoparçacıklarının MWCNT'nin yapısına dahil olduğunu düşündürür. Ayrıca, nanokompozitlerin elektriksel iletkenlik sonuçlarıyla EDS analiz sonuçları bir birleriyle tutarlıdır. R-TEM sonuçlarına göre daha homojen bir şekilde modifiye MWCNT kanallarına $\mathrm{CuO}$ nanoparçacıklarının enkapsüle edilmesi iyi dağılabilirlikle sonuçlanmış ve buna bağlı olarak nanokompozitlerin kendi içerisinde elektriksel iletkenlik değeri artmıştır.

\section{Teşekkür}

Bu çalışma, Hitit Üniversitesi Bilimsel Araştırma Projesi, Türkiye [Proje no: MUH19002.18.002] tarafindan desteklenmiştir.

\section{Kaynakça}

[1] N. Salah, A. Alshahrie, M.S. Abdel-wahab, N.D. Alharbi, Z.H. Khan, Carbon nanotubes of oil fly ash integrated with ultrathin $\mathrm{CuO}$ nanosheets as effective lubricant additives, Diam. Relat. Mater. $78 \quad$ (2017) 97-104. https://doi.org/10.1016/j.diamond.2017.08.010.

[2] J. Yi, D. Fang, L. Li, R. Bao, P. Liu, A facile synthesis of $\mathrm{CNTs} / \mathrm{Cu} 2 \mathrm{O}-\mathrm{CuO}$ heterostructure composites by spray pyrolysis and its visible light responding photocatalytic properties, Adv. Powder Technol. 29 (2018) 2027-2034. https://doi.org/10.1016/j.apt.2018.05.009.

[3] A. Chinnappan, D. Ji, C. Baskar, X. Qin, S. Ramakrishna, 3Dimensional MWCNT/CuO nanostructures use as an electrochemical catalyst for oxygen evolution reaction, J. Alloys Compd. $735 \quad$ (2018) 2311-2317. https://doi.org/10.1016/j.jallcom.2017.11.390.

[4] D. Saravanakkumar, H.A. Oualid, Y. Brahmi, A. Ayeshamariam, M. Karunanaithy, A.M. Saleem, K. Kaviyarasu, S. Sivaranjani, M. Jayachandran, Synthesis and characterization of $\mathrm{CuO} / \mathrm{ZnO} / \mathrm{CNTs}$ thin films on copper substrate and its photocatalytic applications, OpenNano. 4 (2019) 100025. https://doi.org/10.1016/j.onano.2018.11.001.

[5] M.Q. Tran, C. Tridech, A. Alfrey, A. Bismarck, M.S.P. Shaffer, Thermal oxidative cutting of multi-walled carbon nanotubes, Carbon N. Y. 45 (2007) 2341-2350. https://doi.org/10.1016/j.carbon.2007.07.012.

[6] H. Tanabi, M. Erdal, Effect of CNTs dispersion on electrical, mechanical and strain sensing properties of CNT/epoxy nanocomposites, Results Phys. 12 (2019) 486-503. https://doi.org/10.1016/j.rinp.2018.11.081.

[7] S. Maity, S. Das, D. Sen, K.K. Chattopadhyay, Tailored CuO nanostructures decorated amorphous carbon nanotubes hybrid for efficient field emitter with theoretical validation, $\begin{array}{lllll}\text { Carbon } & \text { N. } & \text { Y. } & 127 & \text { (2018) 510-518. }\end{array}$ https://doi.org/10.1016/j.carbon.2017.11.018.

[8] Y. Zhao, M. Ikram, J. Zhang, K. Kan, H. Wu, W. Song, L. Li, $\mathrm{K}$. Shi, Outstanding gas sensing performance of CuO-CNTs nanocomposite based on asymmetrical schottky junctions, Appl. Surf. Sci. 428 (2018) 415-421. https://doi.org/10.1016/j.apsusc.2017.09.173.

[9] P. Cui, A.J. Wang, Synthesis of CNTs/CuO and its catalytic performance on the thermal decomposition of ammonium perchlorate, J. Saudi Chem. Soc. 20 (2016) 343-348. https://doi.org/10.1016/j.jscs.2014.09.010.

[10]M. Kierkowicz, E. Pach, A. Santidrián, S. Sandoval, G. Gonçalves, E. Tobías-Rossell, M. Kalbáč, B. Ballesteros, G. Tobias, Comparative study of shortening and cutting strategies of single-walled and multi-walled carbon nanotubes assessed by scanning electron microscopy, Carbon $\begin{array}{llll}\text { N. } & \text { Y. } & 139 & \text { (2018) }\end{array}$ https://doi.org/10.1016/j.carbon.2018.06.021.

[11]C. Wang, S. Guo, X. Pan, W. Chen, X. Bao, Tailored cutting of carbon nanotubes and controlled dispersion of metal nanoparticles inside their channels, J. Mater. Chem. 18 (2008) 5782-5786. https://doi.org/10.1039/b811560e.

[12]F. Boran, Encapsulation of $\mathrm{CuO}$ nanoparticles inside the channels of the multi-walled carbon nanotubes functionalized 
with thermal stress, Diam. Relat. Mater. 114 (2021) 108306. https://doi.org/10.1016/j.diamond.2021.108306.

[13]S. Morariu, M. Bercea, M. Teodorescu, M. Avadanei, Tailoring the properties of poly(vinyl alcohol)/poly(vinylpyrrolidone) hydrogels for biomedical applications, Eur. Polym. J. 84 (2016) 313-325. https://doi.org/10.1016/j.eurpolymj.2016.09.033.

[14]F. Boran, S. Çetinkaya, M. Karakışla, M. Saçak, Synthesis and characterization of poly(o-toluidine)/kaolinite conductive composites for humidity and temperature sensing, Pamukkale Univ. J. Eng. Sci. 24 (2018) 1278-1283. https://doi.org/10.5505/pajes.2017.94557.

[15]F. Boran, S. Çetinkaya, D. Anaklı, M. Karakışla, M. Saçak, Geliştirilmiş elektrik iletkenliğine sahip POT/Na-Feldispat iletken kompozitlerinin sentezlenmesi ve karakterizasyonu, Dicle Üniversitesi Mühendislik Fakültesi Mühendislik Derg. 8 (2017) 901-910. https://dergipark.org.tr/download/article-file/445867.

[16]D. Candemir, F. Boran, Size controllable synthesis and characterization of cuo nanostructure, in: Mater. Sci. Forum, 2018: pp. 98-103. https://doi.org/10.4028/www.scientific.net/MSF.915.98.

[17]Y.S. Jun, J.G. Um, G. Jiang, A. Yu, A study on the effects of graphene nano-platelets (GnPs) sheet sizes from a few to hundred microns on the thermal, mechanical, and electrical properties of polypropylene (PP)/GnPs composites, Express Polym. Lett. $12 \quad$ (2018) 885-897. https://doi.org/10.3144/expresspolymlett.2018.76.

[18]H. Veisi, B. Karmakar, T. Tamoradi, S. Hemmati, M. Hekmati, M. Hamelian, Biosynthesis of $\mathrm{CuO}$ nanoparticles using aqueous extract of herbal tea (Stachys Lavandulifolia) flowers and evaluation of its catalytic activity, Sci. Rep. 11 (2021) 1-13. https://doi.org/10.1038/s41598-021-81320-6.

[19]S. Barua, P. Chattopadhyay, M.M. Phukan, B.K. Konwar, N. Karak, Hyperbranched epoxy/MWCNT-CuO-nystatin nanocomposite as a high performance, biocompatible, antimicrobial material, Mater. Res. Express. 1 (2015). https://doi.org/10.1088/2053-1591/1/4/045402.

[20]M. Nazim, A.A.P. Khan, A.M. Asiri, J.H. Kim, Exploring Rapid Photocatalytic Degradation of Organic Pollutants with Porous $\mathrm{CuO}$ Nanosheets: Synthesis, Dye Removal, and Kinetic Studies at Room Temperature, ACS Omega. 6 (2021) 2601-2612. https://doi.org/10.1021/acsomega.0c04747.

[21]J. Zhao, S. Ge, D. Pan, Q. Shao, J. Lin, Z. Wang, Z. Hu, T. $\mathrm{Wu}, \mathrm{Z}$. Guo, Solvothermal synthesis, characterization and photocatalytic property of zirconium dioxide doped titanium dioxide spinous hollow microspheres with sunflower pollen as bio-templates, J. Colloid Interface Sci. 529 (2018) 111121. https://doi.org/10.1016/j.jcis.2018.05.091.

[22]A.S. Keiteb, E. Saion, A. Zakaria, N. Soltani, Structural and Optical Properties of Zirconia Nanoparticles by Thermal Treatment Synthesis, J. Nanomater. 2016 (2016) 1-6. https://doi.org/10.1155/2016/1913609.

[23]M.M. Hussain, A.M. Asiri, M.M. Rahman, A non-enzymatic electrochemical approach for L-Lactic acid sensor development based on CuO.MWCNT nanocomposites modified with a nafion matrix, New J. Chem. 44 (2020) 9775-9787. https://doi.org/10.1039/D0NJ01715A.

[24]M. Arfan, D.N. Siddiqui, T. Shahid, Z. Iqbal, Y. Majeed, I. Akram, Noreen, R. Bagheri, Z. Song, A. Zeb, Tailoring of nanostructures: $\mathrm{Al}$ doped $\mathrm{CuO}$ synthesized by compositehydroxide-mediated approach, Results Phys. 13 (2019) 102187. https://doi.org/10.1016/j.rinp.2019.102187.
[25]A. Kumar, D. Kumar, G. Pandey, Characterisation of Hydrothermally Synthesised Cuo Nanoparticles at Different pH, J. Technol. Adv. Sci. Res. J. 2 (2016) 166-169. https://doi.org/10.14260/jtasr/2016/29.

[26]I.Y. Erdoğan, Ö. Güllü, Optical and structural properties of $\mathrm{CuO}$ nanofilm: Its diode application, J. Alloys Compd. 492 (2010) $378-383$. https://doi.org/10.1016/j.jallcom.2009.11.109.

[27]Ş. Baturay, Structural and Optical Properties of Sb Doped CuO Films, Acad. Platf. J. Eng. Sci. 8 (2020) 84-89. https://doi.org/10.21541/apjes.605822.

[28]X. Zhang, D. Zhang, X. Ni, H. Zheng, Optical and electrochemical properties of nanosized $\mathrm{CuO}$ via thermal decomposition of copper oxalate, Solid. State. Electron. 52 (2008) 245-248. https://doi.org/10.1016/j.sse.2007.08.009.

[29]W. Jia, E. Reitz, H. Sun, B. Li, H. Zhang, Y. Lei, From $\mathrm{Cu} 2(\mathrm{OH}) 3 \mathrm{Cl}$ to nanostructured sisal-like $\mathrm{Cu}(\mathrm{OH}) 2$ and $\mathrm{CuO}$ : Synthesis and characterization, J. Appl. Phys. 105 (2009). https://doi.org/10.1063/1.3097286.

[30]J.A. Rudd, E. Kazimierska, A.R. Barron, E. Andreoli, C.E. Gowenlock, A.M. Al-Enizi, V. Gomez, Solvent-free microwave-assisted synthesis of tenorite nanoparticledecorated multi-walled carbon nanotubes, J. Mater. Sci. Technol. (2019). https://doi.org/10.1016/j.jmst.2019.01.002.

[31]Y. Zhao, M. Ikram, J. Zhang, K. Kan, H. Wu, W. Song, L. Li, $\mathrm{K}$. Shi, Outstanding gas sensing performance of CuO-CNTs nanocomposite based on asymmetrical schottky junctions, Appl. Surf. Sci. $428 \quad$ (2018) 415-421. https://doi.org/10.1016/j.apsusc.2017.09.173.

[32]C.H. Lau, R. Cervini, S.R. Clarke, M.G. Markovic, J.G. Matisons, S.C. Hawkins, C.P. Huynh, G.P. Simon, The effect of functionalization on structure and electrical conductivity of multi-walled carbon nanotubes, J. Nanoparticle Res. 10 (2008) 77-88. https://doi.org/10.1007/s11051-008-9376-1.

[33]G. Ran, X. Chen, Y. Xia, Electrochemical detection of serotonin based on a poly(bromocresol green) film and Fe3O4 nanoparticles in a chitosan matrix, RSC Adv. 7 (2017) 1847-1851. https://doi.org/10.1039/c6ra25639b.

[34]A. Manjunath, M. Irfan, K.P. Anushree, K.M. Vinutha, N. Yamunarani, Synthesis and Characterization of $\mathrm{CuO}$ Nanoparticles and $\mathrm{CuO}$ Doped PVA Nanocomposites, Adv. Mater. Phys. Chem. $06 \quad$ (2016) 263-273. https://doi.org/10.4236/ampc.2016.610026. 